Original papers

\title{
Cost-effective autonomous sensor for the long-term monitoring of water electrical conductivity of crop fields
}

\author{
Ernesto Serrano-Finetti*, Carles Aliau-Bonet, Oscar López-Lapeña, Ramon Pallàs-Areny \\ Instrumentation, Sensors and Interfaces Group, Universitat Politècnica de Catalunya - BarcelonaTech, C. Esteve Terradas, 7, 08860 Castelldefels (Barcelona), Spain
}

\section{A R T I C L E I N F O}

\section{Keywords:}

Water quality

Electrical conductivity

Complex electrical impedance

Autonomous sensor

Crop field

\begin{abstract}
A B S T R A C T
Salinity is a key parameter determining water quality. In agriculture, irrigation water with high salinity levels impacts plant growth and yield negatively hence there is a need to monitor it and irrigate with fresh water whenever salinity surpasses the tolerance threshold of a cultivar. Electrical conductivity (EC) of water is usually measured periodically instead of salinity because of its practicality. This work describes a low-cost low-power (and yet inexpensive) autonomous sensor prototype that is able to compute continuously the EC of water from complex electrical impedance measurements based on synchronous sampling, a technique that lowers cost and power consumption. The sensor is easy to assemble and has been verified in the lab for an EC range from $0.35 \mathrm{dS} \mathrm{m}^{-1}$ to $6.18 \mathrm{dS} \mathrm{m}^{-1}$, showing a maximal deviation of $\pm 0.03 \mathrm{dS} \mathrm{m}^{-1}$ from the readings of a commercial reference EC meter. The sensor has also been installed in a rice paddy and left unattended for a whole cultivation season (107 days). The maximum deviation observed during this field test is $\pm 0.14 \mathrm{dS} \mathrm{m}^{-1}$, which is good enough to detect high salinity levels.
\end{abstract}

\section{Introduction}

High salinity levels in irrigation water negatively affects plant growth (Hanson et al., 2006) hence it is considered a key parameter in determining water quality. Dissolved salts translate into ions $\mathrm{Na}^{+}$and $\mathrm{Ca}^{+}$for example) that make water more electrically conductive. Therefore, a practical approach to measure water salinity is by measuring its electrical conductivity (EC). Plants exhibit some tolerance to water salinity, meaning that the crop yield remains almost unaffected below a given salinity threshold and decreases above it. The three most important crops in the world are wheat, rice and maize. Of these, rice is the most salt-sensitive with a yield decrease threshold of $1.9 \mathrm{dS} \mathrm{m}^{-1}$, and many rice cultivars suffer a $50 \%$ reduction in growth at concentration of salts about $7.4 \mathrm{dS} \mathrm{m}^{-1}$ (Grattan et al., 2002). In other crops, yield potential is decreased differently as shown in (Grattan, 2002). Salinity can result from the use of salty water to irrigate fields, from soil salinity, or from both. When the problem is soil salinity, common practice is to irrigate the fields with quality water (EC below $0.75 \mathrm{dS} \mathrm{m}^{-1}$ ) to leach ("wash away") salt from the root zone. On the other hand, if the problem is the salinity of irrigation water (i.e. river water), a common approach is to pump water from upriver freshwater reservoirs rather than from the river. This poses the problem of continuously measuring EC to allow for optimal solutions in terms of water use and energy expenditure (water pumping).

Traditionally, growers suffering these problems use handheld meters for spot measurements but their cost is in the order of hundreds of euros. Moreover, those meters are not prepared for autonomous operation and probes suffer wear or damage from large submersion periods, thus resulting in tedious recalibration procedures and even probe replacement. To overcome these limitations, unattended continuous water measurement by means of autonomous sensor (AS) nodes has been proposed as in (López Riquelme et al., 2009) and (Nam et al., 2017). Moreover, it is expected that, in the future, less fresh water will be available and of a lower quality. Optimal strategies for water management, not only in agriculture, will require continuous and on-line knowledge of water quality (Zia et al., 2013). The solution will come in the form of inexpensive, robust and accurate AS (Zhuiykov, 2012).

An AS that is intended to work unattended in field conditions during a whole season should meet some key requirements like ruggedness, low power consumption, low cost and ease of maintenance at the end of the season. Thus the AS design (both electrical and mechanical) should be kept as simple as possible but without sacrificing accuracy. Using two galvanic electrodes is quite common in EC meters and usually implies the simplest circuitry possible. The impedance between both electrodes is measured, which depends on the series combination of the electrical resistance of water $\left(R_{\mathrm{w}}\right)$ and the electrode impedance $\left(Z_{\mathrm{p}}\right)$ due

\footnotetext{
* Corresponding author.

E-mail addresses: ernesto.serrano@upc.edu (E. Serrano-Finetti), oscar.lopez@upc.edu (O. López-Lapeña), ramon.pallas@upc.edu (R. Pallàs-Areny).
} 


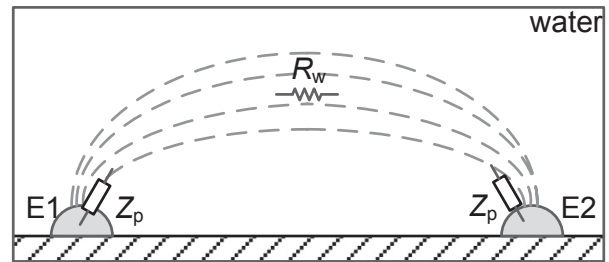

Fig. 1. Conductivity cell with two electrodes (E1 and E2) showing the current distribution between them. $Z_{\mathrm{p}}$ is the polarization impedance associated with the electrode/electrolyte interface and $R_{\mathrm{w}}$ is the resistance of water due to its EC.

to the polarization of the electrode/electrolyte interface (Fig. 1). A high-frequency sinewave is used to cancel out the effects of the series $Z_{\mathrm{p}}$. However, deviations may still occur if there is prolonged electrode corrosion, as is the case of continuous EC measurement.

Concerning circuitry, using a minimum number of electronic components is also a desirable feature. To recover the impedance information, the sinewave (carrier) is injected and the response comes in the form of an amplitude-modulation (AM) waveform. Analog demodulators are thus used to recover the impedance measurements (Min et al., 2000). However, their use can be obviated by using a digitizing scheme known as quadrature synchronous sampling (QSS) (PallàsAreny and Webster, 1993). This technique is similar to band-pass sampling but requires taking two samples of the sinusoidal response signal $90^{\circ}$ (a quarter of the carrier period) apart, from which it is easy to recover the in-phase and quadrature components of the impedance, that is, it is capable of measuring the complex impedance. It needs low-cost circuitry, mostly a microcontroller unit (MCU) with embedded analogto-digital (ADC) and digital-to-analog (DAC) converters -common nowadays and available at low cost- and a current-to-voltage converter, instead of the of two dedicated analog demodulators used by most impedance meters. There also exist integrated circuits (IC) such as the AFE4300 (Texas Instruments, Inc.) or the AD5933 (Analog Devices, Inc.) that offer complete analog front-ends to measure electrical impedance, but they might require additional circuitry if the impedance is to be measured with low uncertainty (Sanchez et al., 2013; Seoane et al., 2008).

In this work, we propose the design and implementation of a rugged, cost-effective, low-power consumption EC autonomous sensor based on QSS able to monitor water salinity continuously without supervision. To reduce the manufacture costs, the sensor housing and conductivity cell are built from polyvinyl chloride (PVC) pipes and fittings and the electrodes are built from grade A4 stainless steel nuts and bolts. $R_{\mathrm{w}}$ is derived from the real part of the measured complex impedance. Also, water temperature is measured to compute the equivalent $\mathrm{EC}$ of water at $25^{\circ} \mathrm{C}$.

\section{Quadrature synchronous sampling}

Measuring an electrical impedance

$Z_{\mathrm{x}}=R+j X=\left|Z_{\mathrm{x}}\right| e^{j \theta_{\mathrm{x}}}$

by applying a sinusoidal voltage of known amplitude (the carrier signal) $v(t)=V \cos \left(2 \pi f_{\mathrm{c}} t\right)$ involves measuring the current response $i(t)$ through it,

$$
\begin{aligned}
i(t)=I \cos \left(2 \pi f_{\mathrm{c}} t-\theta_{\mathrm{x}}\right)= & \frac{V}{\left|Z_{\mathrm{x}}\right|} \cos \left(\theta_{\mathrm{x}}\right) \cos \left(2 \pi f_{\mathrm{c}} t\right)+\frac{V}{\left|Z_{\mathrm{x}}\right|} \sin \left(\theta_{\mathrm{x}}\right) \sin \left(2 \pi f_{\mathrm{c}} t\right) \\
& =P \cos \left(2 \pi f_{\mathrm{c}} t\right)+Q \sin \left(2 \pi f_{\mathrm{c}} t\right)
\end{aligned}
$$

where $P$ and $Q$ are the amplitude of the in-phase and quadrature components respectively. Therefore, since the excitation amplitude $V$ is known, $Z_{x}$ can be computed as

$\left|Z_{\mathrm{x}}\right|=V / \sqrt{P^{2}+Q^{2}}$ $\theta_{\mathrm{x}}=\tan ^{-1}(Q / P)$

$P$ and $Q$ can be recovered from samples of $i(t)$ at times

$t_{R}=n T_{\mathrm{c}} / 2$

and

$t_{X}=(n+m) T_{\mathrm{c}} / 2+T_{\mathrm{c}} / 4$

where $T_{\mathrm{c}}=1 / f_{\mathrm{c}}$ is the carrier period and $n$ is the sample number, $n$ and $m$ being integers (Pallàs-Areny and Webster, 1993). All timing is synchronized with $v(t)$. In this way, for $n=m=0, t_{\mathrm{R}}$ and $t_{\mathrm{X}}$ are $T_{\mathrm{c}} / 4$ apart, yielding $s_{1}=P$ and $s_{2}=Q$.

The range of interest for water $\mathrm{EC}$ at $25^{\circ} \mathrm{C}\left(\mathrm{EC}_{25}\right)$ considered is from $0.5 \mathrm{dS} \mathrm{m}^{-1}$ to $5.0 \mathrm{dS} \mathrm{m}^{-1}$. For some crops, EC values between $1.0 \mathrm{dS} \mathrm{m}^{-1}$ and $2.0 \mathrm{dS} \mathrm{m}^{-1}$ are more relevant because they set the trigger point to initiate some action (i.e. irrigation with fresh, low salinity water) to avoid a decrease in crop yield. The target resolution is $0.01 \mathrm{dS} \mathrm{m}^{-1}$ for the extended EC range from $0.4 \mathrm{dS} \mathrm{m}^{-1}$ to $6.0 \mathrm{dS} \mathrm{m}^{-1}$, which results from the specified EC range and a water temperature $\left(T_{\mathrm{w}}\right)$ range from $15{ }^{\circ} \mathrm{C}$ to $35^{\circ} \mathrm{C}$. The temperature resolution is set at $0.1^{\circ} \mathrm{C}$. For the specified EC resolution, the required dynamic range if no offset correction circuitry is used is $\mathrm{DR}=(6.0-0.0) / 0.01=600$, which asks for 10 bit minimum. The MCU selected is the MSP430FG4618 (Texas instruments Inc.), featuring a 12-bit ADC, hence allowing room for additional uncertainty factors, and an internal sample \& hold $(\mathrm{S} / \mathrm{H})$ circuitry. This MCU also features low power consumption, low-voltage operation and a maximum CPU clock frequency of $8 \mathrm{MHz}$.

The modulus and phase of $Z_{\mathrm{x}}$ can thus be computed as in (3) an (4) and $R$ and $X$ will be

$R=\left|Z_{\mathrm{x}}\right| \cos \left(\theta_{\mathrm{x}}\right)$

$X=\left|Z_{\mathrm{x}}\right| \sin \left(\theta_{\mathrm{x}}\right)$

When $m \neq 0$, the samples for $P$ and $Q$ belong to different periods of the carrier signal ( $s_{1}$ and $s_{2}{ }^{\prime}$ in Fig. 2 ). This relaxes the timing requirements whenever the conversion time $t_{\text {conv }}$ of the ADC is comparable to the period of the carrier. However, this requires that $Z_{\mathrm{x}}$ does not change over time or that it is changing below the ADC's resolution between those sampling times. This timing scheme is also beneficial if $i$ ( $t$ ) has an offset because if the samples coming from adjacent halfperiods $s_{3}$ and $s_{4}$ are phase-reversed versions of $s_{1}$ and $s_{2}$, subtracting from each other cancels the offset.

\section{Sensor design}

The proposed AS comprises a MCU with an embedded ADC, an analog front-end (AFE) that implements the two-electrode impedance measurement technique, a direct interface circuit that measures the temperature with a resistive sensor (Pt1000) using the direct sensor-

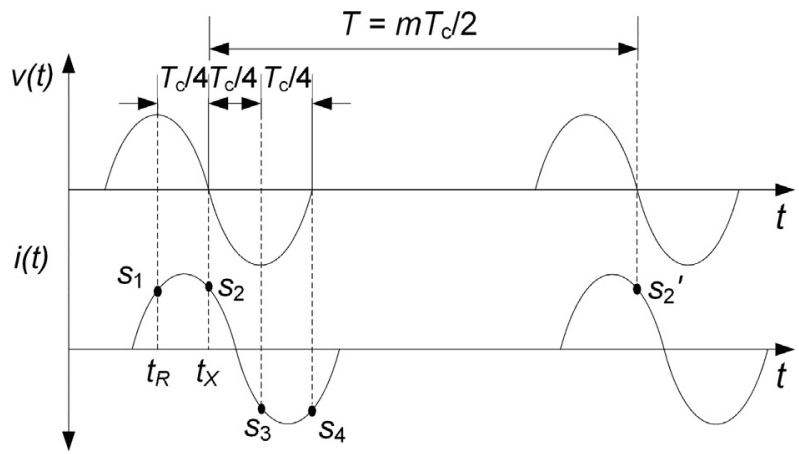

Fig. 2. Waveforms of the applied voltage and the detected current in impedance measurement showing the sampling times $t_{R}$ and $t_{X}$ and other equivalent sampling times that contain the same information about the real and imaginary parts of the impedance. 


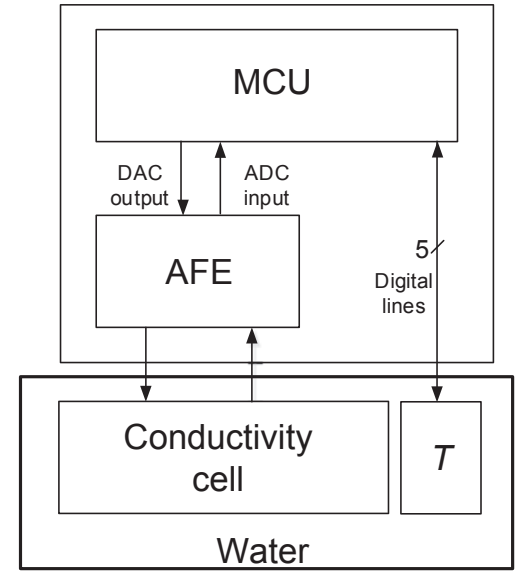

Fig. 3. Block diagram showing the architecture of the EC autonomous.

microcontroller interface (DSMI) approach (Reverter and Pallàs-Areny, 2005) and a conductivity cell. Fig. 3 shows a block diagram of the proposed architecture. The electronic circuitry comprises an MCU, and AFE and a resistive temperature sensor (RTD).

A key difference compared to other EC handheld or autonomous sensors is the implementation of the QSS technique explained in Section 2 , hence an unknown impedance can be measured without using analog demodulators or Fast Fourier transforms as in the AD5933, saving board space, cost and power consumption as explained later. On the other hand, the conductivity cell consists of the housing of the system where two electrodes are inserted and a volume constrainer to increase the cell factor, as explained in Section 3.5. The system is powered from three AAA 950-mAh Ni-MH (3.6 V total) rechargeable batteries that supply $3.3 \mathrm{~V}$ to the measurement circuitry and the MCU by means of a voltage regulator.

In a two-electrode measurement system, the electrode polarization impedance $Z_{\mathrm{p}}$ appears in series with the water resistance $R_{\mathrm{w}}$, thus becoming a major source of measurement deviations. Using a high frequency sine wave mitigates this problem. For this design, preliminary tests with off-the-shelf instruments established that working frequencies around $100 \mathrm{kHz}$ are high enough. Therefore, the measured $Z_{\mathrm{x}}$ is assumed to be basically due to water resistance $R_{\mathrm{w}}$.

\subsection{Impedance measurement circuitry}

Implementing QSS with MCUs that embed an ADC and a DAC ensures synchronization between signal generation and acquisition because their clock source is the same. Fig. 4 shows a block diagram of the impedance measurement system showing the MCU and the AFE sections. The MCU generates the excitation signal, acquires the response and process the sampled values. The AFE involves: (1) Antialiasing filters for both the DAC and the ADC; (2) Buffering of the filtered voltage stimulus in order to avoid voltage loading effects between the DAC, the filter and $Z_{\mathrm{x}}$; and (3) Current-to-voltage conversion (I/V) of

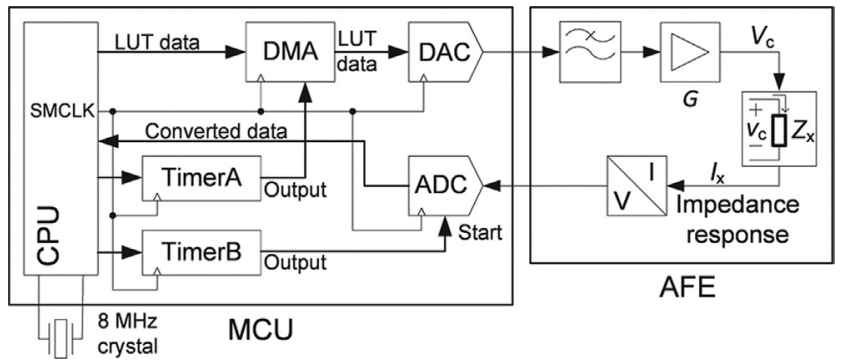

Fig. 4. Block diagram of the impedance measurement system that implements QSS based on an MCU with embedded ADC and DAC, and a simple AFE. the impedance current response by means of a transimpedance amplifier (TIA).

Any synchronization issues between the DAC and the ADC inside the MCU will introduce measurement uncertainty in QSS. To prevent this, both the 12-bit ADC and DAC clock inputs are fed by two timers (Timer A and Timer B) which are started simultaneously and whose clock source is the MCU's sub-main clock (SMCLK), which is a copy of the main clock (MCLK). SMCLK is sourced from an $8 \mathrm{MHz}$ quartz crystal $\left(f_{\text {MCLK }}=8 \mathrm{MHz}\right)$. Sine waves are generated from a look-up table (LUT) of length $L=16$, that is, it has 16 sine wave values per cycle. For fast signal generation, LUT data is transferred to the DAC using the directmemory access (DMA) peripheral. According to the manufacturer, each transfer cycle can take up to five MCLK cycles hence Timer A cannot be assumed to always trigger the DMA in less than this time. This sets a theoretical maximum frequency of $100 \mathrm{kHz}$ for the sine wave with a MCLK frequency of $8 \mathrm{MHz}$. However, previous experiments suggest to work with a transfer cycle of six MCLK cycles, hence the sine wave frequency will be $83.3 \mathrm{kHz}$.

Fig. 5 shows the details of the AFE circuitry designed which is supplied with the same MCU supply voltage. Using $L=16$ implies coarse discretization of the sine wave hence some harmonic distortion is expected. For a carrier frequency $f_{\mathrm{c}}$, the DAC output frequency spectrum will exhibit images (harmonics) at $(k \times L \pm 1) f_{\text {c }}$, (where $k$ is any integer) and which amplitudes follow a $\operatorname{sinc}\left(\pi f / f_{\mathrm{c}}\right)$ attenuation function. The ensuing low-pas filter (LPF) provides further attenuation. A $720 \mathrm{kHz}$ cutoff frequency reduces the experimental harmonic distortion to about $5 \%$. The compensation of the systematic deviation introduced by this distortion is explained later.

The DAC amplitude was adjusted in order to yield $100 \mathrm{mV}$ at the LPF output. This filtered sine wave voltage is buffered by OA1a to avoid voltage loading effect, and then applied to the cell impedance, $Z_{\mathrm{x}}$. The current response $i(t)$ from $Z_{\mathrm{x}}$ is further converted to a voltage by means of OA1b configured as a TIA and biased to $\mathrm{V}_{\mathrm{CC}} / 2$ by means of the DAC1 output, thus saving space and cost. OA1a and OA1b are the dual lowvoltage OPA2355 op amp (Texas Instruments Inc.) which exhibit railto-rail input/output capability and a gain-bandwidth product of $200 \mathrm{MHz}$. During AFE tests on $R C$ networks that emulate a Randles cell, large high frequency transients appeared as is normally the case in transimpedance amplifiers (Aliau-Bonet and Pallàs-Areny, 2013). This issue was mitigated with a shunt capacitor $C_{\mathrm{f}}=22 \mathrm{pF}$ (Fig. 5), which reduced the bandwidth to about $2 \mathrm{MHz}$.

From Section 3.4, the cell constant is $K_{\text {cell }}=0.80 \mathrm{~cm}^{-1}$, that allows an $R_{\mathrm{w}}$ range from $2000 \Omega$ to $133 \Omega$. $i(t)$ will thus be in the range from $50 \mu \mathrm{A}$ to $752 \mu \mathrm{A}$ for an applied voltage of $100 \mathrm{mV}$. $R_{\text {ref }}$ was set at $3.9 \mathrm{k} \Omega$ in order to have a $1 \mathrm{~V}$ amplitude sine wave at $v_{\mathrm{o}}(t)$ for EC $\approx 2.0 \mathrm{dS} / \mathrm{m}$ which is the alarm threshold selected. Finally, the output amplitude of OA1b relates to $\left|Z_{\mathrm{x}}\right|$ according to

$V_{\mathrm{o}}=-R_{\mathrm{f}} V /\left|Z_{\mathrm{X}}\right|$

where $V$ is the $100 \mathrm{mV}$ amplitude of the filtered sine wave.

As long as all analog processing blocks (antialiasing filters, $\mathrm{S} / \mathrm{H}$ circuitry, TIA) behave linearly, they will mainly contribute systematic gain and phase deviations from the ideal behavior. A simple approach to compensate them is to calibrate with a reference impedance such as a precision resistor $\left(R_{\text {cal }}\right)$ previous to each measurement.

\subsection{Impedance acquisition strategy, internal calibration and EC calculation}

We use the samples from consecutive carrier half-periods $(m=0)$ hence acquiring four consecutive samples of $V_{\mathrm{o}}$ analogous to $s_{1}$ to $s_{4}$ in Fig. 2. Therefore

$P_{\mathrm{d}}[n]=0.5\left(s_{1}[n]-s_{3}[n]\right)$

$Q_{\mathrm{d}}[n]=0.5\left(s_{2}[n]-s_{4}[n]\right)$

where $\mathrm{d}$ stands for 'digitized' and $n$ is the sample number. $P$ and $P_{\mathrm{d}}$ 


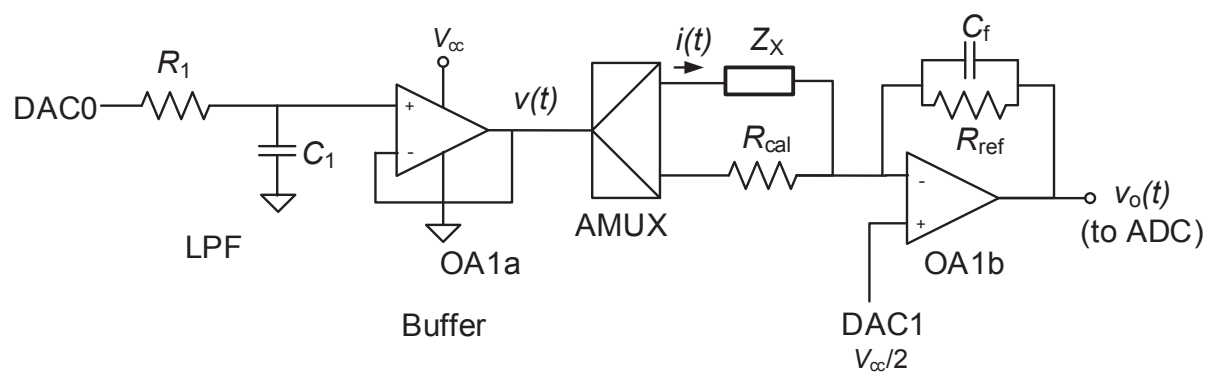

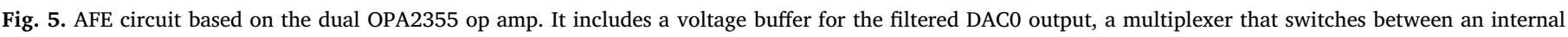
calibration resistor and the conductivity cell and a TIA. DAC1 provides level shifting.

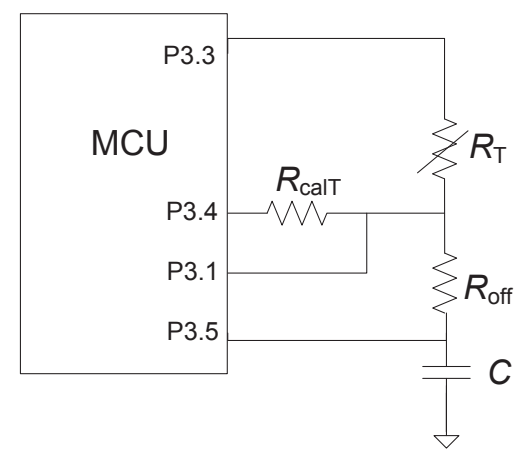

Fig. 6. Resistance measurement by indirect digital conversion based on obtaining a pulse whose duration is proportional to the resistance value $R_{\mathrm{T}}$, which measures water temperature $\left(T_{\mathrm{w}}\right)$.

should not be confused, as the latter is a number straightly computed from the ADC codes $s_{i}$. The same is true for $Q$ and $Q_{\mathrm{d}}$.

In synchronous sampling systems, a high-pass filter precedes the $S$ / $\mathrm{H}$ and a low-pass filter follows it in order to attenuate low-frequency and high-frequency noise and interference (Pallàs-Areny and Webster, 1993). Nevertheless, the subtraction in (10) and (11) has a high-pass filter effect (Oppenheim et al., 1997) hence eliminating any offset error at the output of the TIA, as well as attenuating very low-frequency noise. On the other hand, averaging a number of consecutive samples implements a digital low-pass filter. This way, if $N_{\mathrm{s}}$ samples are taken $T_{\mathrm{c}} / 4$ apart from each other, there will be $N_{\mathrm{s}} / 4 P_{\mathrm{d}}$ and $Q_{\mathrm{d}}$ values that can be averaged to reduce the uncertainty (standard deviation) by a factor of $\sqrt{ }\left(N_{\mathrm{s}} / 4\right)$, at the expense of lengthening the measurement time. These software filters reduce size and the bill of components. In our case, $N_{\mathrm{s}}=128$ hence the total measurement time will be $32 T_{\mathrm{c}}$.

Internal calibration compensates for any gain and phase deviations introduced by the AFE or any permanent deviations from the nominal values of the ADC reference voltage and the carrier amplitude. A precision resistor $R_{\text {cal }}$ replaces $Z_{\mathrm{x}}$ and a number of consecutive samples of $P_{\mathrm{d}}$ and $Q_{\mathrm{d}}$ are acquired and averaged yielding $P_{\mathrm{cal}}$ and $Q_{\mathrm{cal}}$. From them, a calibration magnitude $\left(\left|M_{\mathrm{cal}}\right|\right)$ and phase $\left(\theta_{\mathrm{cal}}\right)$ are computed

$\left|M_{\text {cal }}\right|=\sqrt{P_{\text {cal }}^{2}+Q_{\text {cal }}^{2}}$

$\theta_{\text {cal }}=\tan ^{-1}\left(Q_{\text {cal }} / P_{\text {cal }}\right)$

and stored in the MCU's flash memory. After this calibration stage, the measurement proceeds as follows. An unknown impedance $Z_{x}$ is measured yielding

$|M|=\sqrt{P_{\mathrm{d}}^{2}+Q_{\mathrm{d}}^{2}}$

$\theta=\tan ^{-1}\left(Q_{\mathrm{d}} / P_{\mathrm{d}}\right)$

that are the uncalibrated magnitude and phase. Since $M$ and $M_{\text {cal }}$ are complex numbers, from (9) it follows that

$Z_{\mathrm{x}}=R_{\mathrm{cal}} M_{\mathrm{cal}} / M$ hence

$\left|Z_{\mathrm{x}}\right|=R_{\text {cal }}\left|M_{\text {cal }}\right| /|M|$

$\theta_{\mathrm{x}}=\angle Z_{\mathrm{x}}=\theta_{\text {cal }}-\theta$

In our implementation, $\left|M_{\text {cal }}\right|$ and $\theta_{\text {cal }}$ were estimated by measuring $R_{\text {cal }} 100$ times and then averaging the results. Therefore, the uncertainty in $\left|Z_{\mathrm{x}}\right|$ and $\theta_{\mathrm{x}}$ would not be affected by the uncertainty of $\left|M_{\text {cal }}\right|$ and $\theta_{\text {cal }}$.

Because this is a one-point internal calibration process, $R_{\text {cal }}$ was selected a $\pm 0.05 \%, \pm 10 \mathrm{ppm} /{ }^{\circ} \mathrm{C} 390 \Omega$ resistor, hence very close to the $2 \mathrm{dS} \mathrm{m}^{-1}$ threshold established before. Such a low temperature coefficient yields negligible deviation for the required $20^{\circ} \mathrm{C}$ water temperature span.

\subsection{Temperature measurement}

The DSMI method involves the application of a pulse to an $R C$ network containing a resistive sensor. The charge time of that network is proportional to the resistance of the sensor and the MCU measures it with an internal timer. This means that no ADC nor signal conditioning circuits are necessary, again saving board space, cost and power consumption. Fig. 6 shows the implementation of this technique. Here, the temperature sensor is a Pt1000 and the MCU port internal resistances are considered. Briefly, the method described in (Reverter and PallàsAreny, 2005) consists in taking three time counts -performed with an internal counter- $N_{\text {off }}, N_{\text {CalT }}$ and $N_{\text {T }}$ for three different digital ports settings meant to charge and discharge capacitor $C$. If all output ports have the same internal resistance $R_{\text {out }}$, the corresponding count numbers will be

$N_{\text {off }}=K\left(R_{\text {out }}+R_{\text {off }}\right) C$

$N_{\text {CalT }}=K\left(R_{\text {out }}+R_{\text {off }}+R_{\text {CalT }}\right) C$

$N_{\mathrm{T}}=K\left(R_{\text {out }}+R_{\text {off }}+R_{\mathrm{T}}\right) C$,

and the resistance of the sensor can be calculated by

$R_{\mathrm{T}}=R_{\text {CalT }} \frac{N_{\mathrm{T}}-N_{\text {off }}}{N_{\text {CalT }}-N_{\text {off }}}$

The Pt1000 sensor is assumed to be linear in the measurement range according to the model

$R_{\mathrm{T}}=R_{0}\left[1+\alpha_{0}\left(T_{\mathrm{w}}-0{ }^{\circ} \mathrm{C}\right)\right]$

therefore

$T_{\mathrm{w}}=\frac{1}{\alpha_{0}}\left(\frac{R_{\mathrm{T}}}{R_{0}}-1\right)$.

\subsection{Power consumption}

To reduce power consumption, the AS is held in sleep mode most of the time and is awaken just the time necessary to perform the measurements as explained in Section 3.2. It was deemed adequate to 


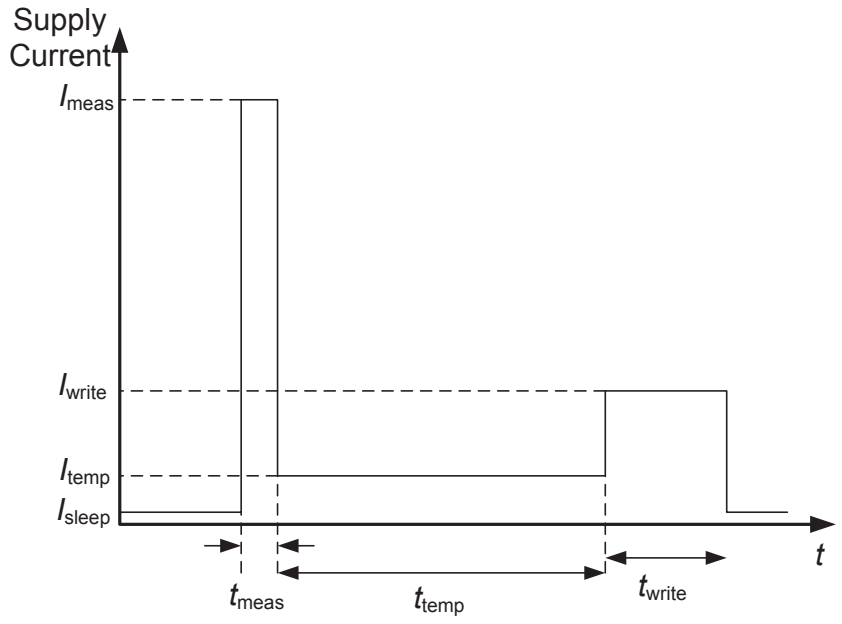

Fig. 7. Supply current during the active mode. The AS performs the impedance (real and imaginary parts) measurement during $t_{\text {meas }}$ ant the temperature measurement during $t_{\mathrm{temp}}$. Finally, it writes the results in the MCU's flash memory during $t_{\text {write }}$. The rest of the time, the AS is in sleep mode and the consumed current is $I_{\text {sleep. }}$.

perform one EC measurement per hour.

Fig. 7 depicts the time course of the supply current in active and in sleep mode. In the active mode the AFE, the MCU core and the embedded ADC and DAC perform a measurement that lasts $t_{\text {meas }}=0.39 \mathrm{~ms}$ and consumes a current $I_{\text {meas }}=11.72 \mathrm{~mA}$. Next, the ADC, the DAC and the AFE are switched off and the AS measures temperature for $t_{\text {temp }}=3.2 \mathrm{~ms}$ and consumes $I_{\text {temp }}=0.94 \mathrm{~mA}$. Finally, only the MCU core and the flash memory peripheral are active and the measurements are written into the MCU's flash memory during $t_{\text {write }}=1 \mathrm{~ms}$ with a current consumption of $I_{\text {write }}=3.88 \mathrm{~mA}$. When in sleep mode, the whole AS consumes $15 \mu \mathrm{A}$. Therefore, the average current consumption is $2.5 \mathrm{~mA}$ during the total active mode time of $4.59 \mathrm{~ms}$. However, the duty cycle is very low $(0.00013 \%)$ hence the active mode current consumption adds only $0.32 \mu \mathrm{A}$ during the $1 \mathrm{~h}$ measurement cycle, making a total average current consumption of $15.32 \mu \mathrm{A}$.

Commercial impedance measurement devices like the AD5933 or the AFE4300 have power-down modes of $5 \mu \mathrm{A}$ and $100 \mu \mathrm{A}$ respectively. However, both of them require an MCU to read and store the measured data (increasing current consumption in sleep mode), and they also require additional circuitry for temperature measurement. On the other hand, the AD5933 has and active mode current consumption in excess of $12 \mathrm{~mA}$ while the AFE4300 only declares a current consumption of about $1 \mathrm{~mA}$ for its embedded ADC but does not provide the consumption estimate of its demodulator stage. Summarizing, the relevant parameter for power consumption is the sleep-mode current, which is quite low in the proposed AS. It should be noted that, in spite of its low power consumption, the proposed AS has low measurement uncertainty as compared to the IC solutions, as explained in Section 1.

\subsection{Conductivity cell and mechanical housing design}

Most commercial EC probes tend to be expensive due to the materials used and, especially, the electrode fabrication processes involved. To minimize costs and to ease the manufacturing process, the proposed EC cell design uses cylindrical PVC pieces that, on the other hand, are rugged enough for field deployment. Fig. 8a shows a picture of the EC cell designed.

The electrodes are two acorn nuts made of grade A4 AISI 316 stainless steel mounted on a fitting hard cap (Fig. 8a) and the mating bolts perform as the electrical terminals. Such nuts are extremely resistant to corrosion and common used in marine applications, which makes them easy-to-get and affordable. Another advantage is that they a

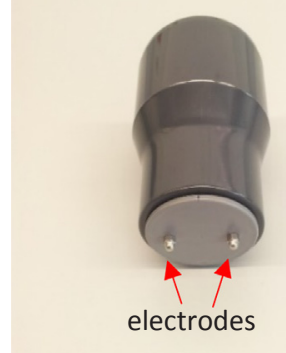

b

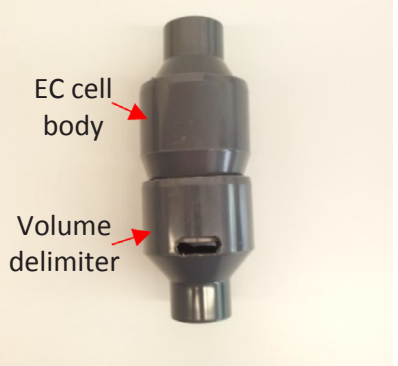

Fig. 8. EC cell: (a) body with the two electrodes, and (b) volume delimiter with two side slits to allow for water flow into the cell.

are easy to clean and replace if deemed necessary.

In this electrode configuration, electric field lines extend beyond the volume between the two electrodes (fringe electric field leading to the so-called edge effects), and this may result in undesired influence of nearby objects or water movement in the volume reached by those fringe electric fields. To avoid this effect, the measurement volume is delimited by a cylindrical PVC shield with some small holes that allow water to freely penetrate and leave that volume (Fig. 8b), but at the same time avoid large water movements near the electrodes. Because the plastic body of the EC cell is an electrical insulator, it confines electric field lines and water outside the cell has negligible influence on the electrical conductivity measured. With this volume delimiter, experimental tests yielded $K_{\text {cell }}=0.80 \mathrm{~cm}^{-1}$ which is good enough for this application.

The PVC shield accepts a tube at its end that facilitates its insertion into the ground. The EC cell body encloses all the electronics necessary to measure EC and store the values to be later recovered by a USB connector. The temperature sensor is glued to the inner face of the fitting cap where the electrodes are mounted. The external face will be in contact with the water hence the sensor will reach the same temperature but with a long time constant. It should be noted that, unlike some commercial EC meters, this AS is designed only for water EC measurements and not for EC soil measurements basically because of the chosen electrode configuration. Soil measurements usually require long, thin electrodes that can be inserted into the soil.

\subsection{Prototype cost}

The housing is made of one $50 \mathrm{~mm}$ PVC end cap and a conical reducer, and the conductivity cell is made of a second conical reducer. They have an approximate cost of $4.80 €$. The electrodes are made of two acorn nuts (M3 DIN 1537, grade A4) and have a cost is $0.15 €$ each. The rest of the needed common mechanical hardware (bolts, nuts, Orings) add up $0.50 €$ to the cost hence the housing cost would be $5.60 €$. The electronic components (active and passive) have an approximate cost of $29.30 €$ (retail prices). Therefore, this prototype AS has a cost of $34.90 €$. Production cost will differ according to the total number of units manufactured.

\section{Experimental setup}

Verification tests in lab conditions and field validation tests have been carried out in order to check the AS performance.

\subsection{Verification tests with saline solutions}

The whole AS was immersed in a saline solution contained in a glass beak (Fig. 9) and whose EC was incrementally increased by adding common salt in small amounts, resulting in a measured EC range from 


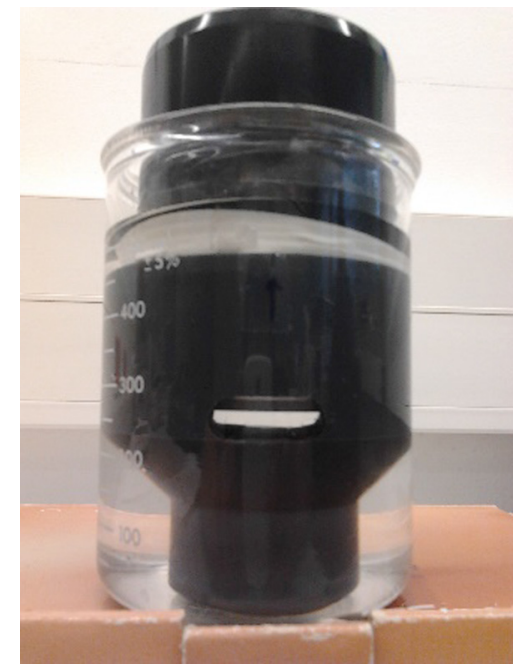

Fig. 9. The autonomous sensor immersed in a saline solution for lab tests.

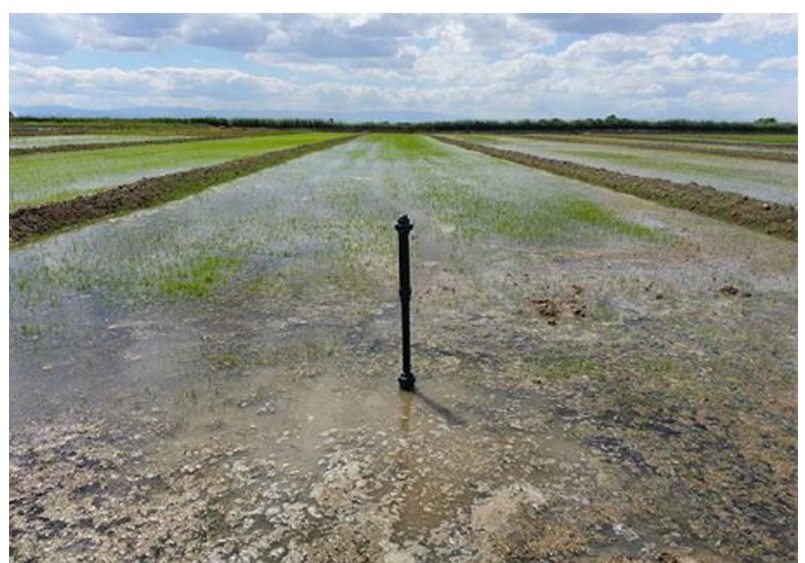

Fig. 10. Installation of the AS in an experimental paddy field at Kalochori, Greece.

$0.351 \mathrm{dS} \mathrm{m}^{-1}$ to $6.18 \mathrm{dS} \mathrm{m}^{-1}$. The sensor measured the real and imaginary parts of the saline solution impedance at $83.3 \mathrm{kHz}$ at room temperature, and the EC value was estimated from the measured real part. The measured temperature and EC values were compared to those obtained with a Multi340i portable conductivity meter (WTW Gmbh). Five consecutive impedance measurements were acquired for each EC measured and their mean value and standard deviation calculated. Reference and sensor measurements were compared through a Bland-

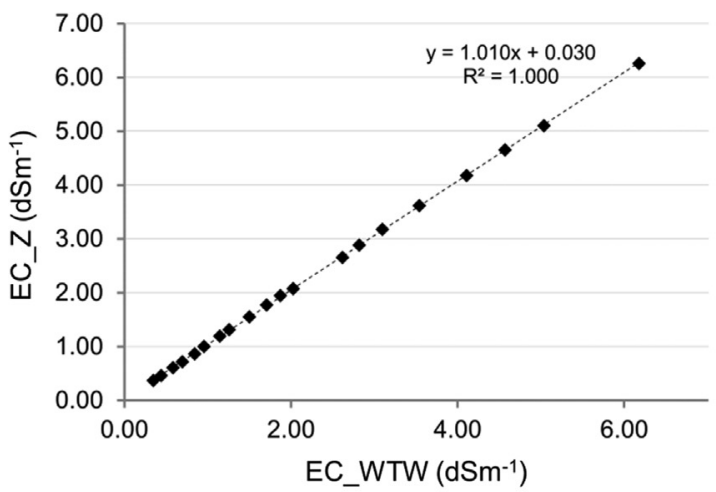

(a)
Table 1

Measured EC values with the WTW conductivity meter and the AS under different temperatures.

\begin{tabular}{|c|c|c|c|c|}
\hline \multicolumn{2}{|l|}{ EC_WTW } & \multicolumn{2}{|l|}{ EC_Z } & \multirow[t]{2}{*}{$\mathrm{EC}_{25} \mathrm{Dev} / \mathrm{dS} \mathrm{m}^{-1}$} \\
\hline $\mathrm{EC}_{25} / \mathrm{dS} \mathrm{m}^{-1}$ & $\mathrm{~T} /{ }^{\circ} \mathrm{C}$ & $\mathrm{EC}_{25} / \mathrm{dS} \mathrm{m}^{-1}$ & $\mathrm{~T} /{ }^{\circ} \mathrm{C}$ & \\
\hline 2.09 & 19.9 & 2.09 & 19.9 & 0.00 \\
\hline 2.09 & 34.7 & 2.10 & 34.8 & -0.01 \\
\hline 0.983 & 19.1 & 0.987 & 19.1 & 0.00 \\
\hline 0.987 & 33.4 & 0.992 & 33.5 & -0.01 \\
\hline 4.05 & 18.3 & 3.93 & 18.3 & 0.12 \\
\hline 4.06 & 34.5 & 3.98 & 34.6 & 0.08 \\
\hline
\end{tabular}

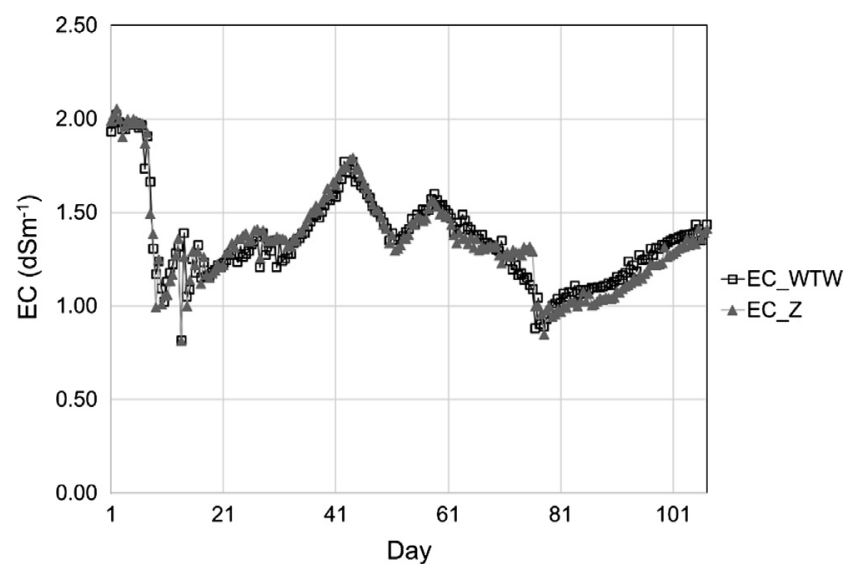

Fig. 12. Daily EC measurements obtained in the field test for 15 weeks by both the AS (EC_Z) and the reference conductivity meter (EC_WTW).

Altman agreement analysis.

To quantify the influence of temperature on impedance measurements, some EC values in the target EC range were measured at $19^{\circ} \mathrm{C}$ and $35^{\circ} \mathrm{C}$.

\subsection{Field tests}

Field experiments were performed in an experimental paddy field that was sowed two weeks before (Fig. 10), and programmed to automatically measure EC and temperature each hour and store the results in the MCU's internal flash memory. The sensor was housed in a long PVC tube that included the power supply, based on a small solar cell, and taller than the rice plants at their maximum growth. This PVC tube was stuck in the field in such a way that the electrodes were about $5 \mathrm{~cm}$ above the ground level.

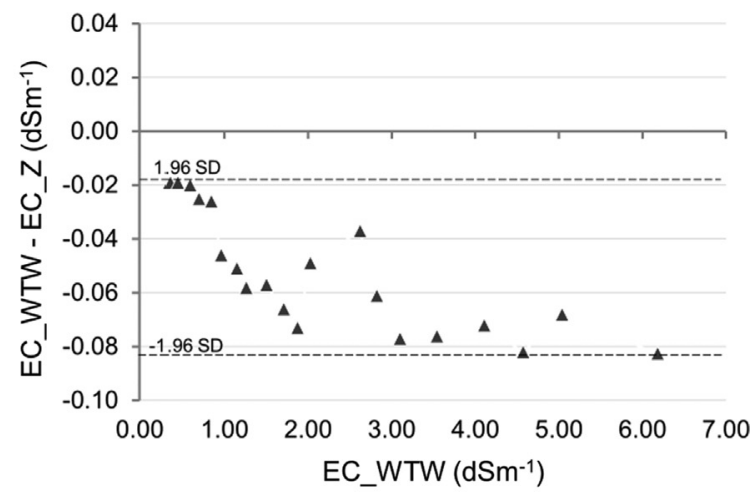

(b)

Fig. 11. Measurement comparison between the AS (EC_Z) and the WTW meter (EC_WTW) for different saline conductivities. (a) Linear regression between EC_Z and EC_WTW, and (b) Evolution of the difference between EC_WTW and EC_Z against the mean value obtained by both the sensor and the WTW meter. 


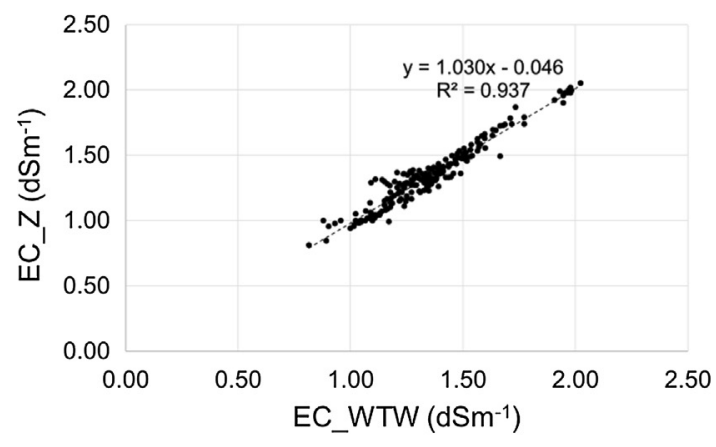

(a)

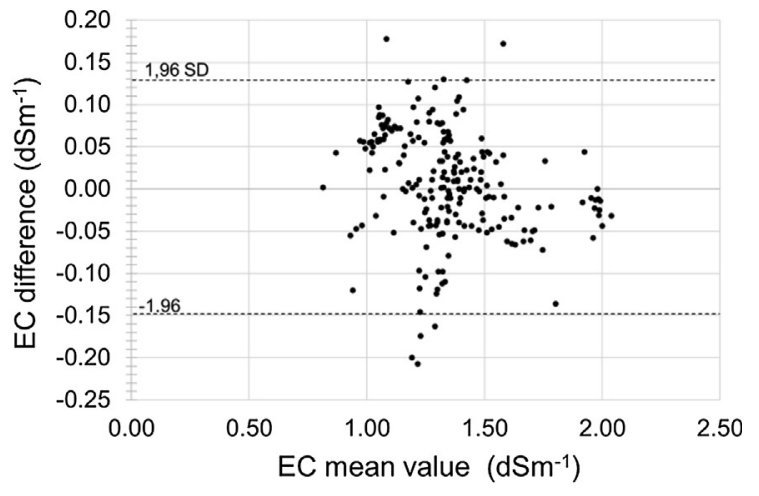

(b)

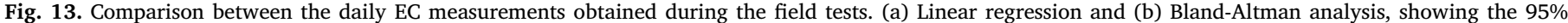
confidence interval.

EC was also manually measured by the station's staff twice a day with a VARIO Cond handheld EC meter (WTW Gmbh) at about the same time $( \pm 5 \mathrm{~min})$ and near $(5-10 \mathrm{~cm})$ the AS. The meter's probe was placed very close to the sensor electrodes. The meter has a temperature uncertainty of $\pm 0.5^{\circ} \mathrm{C}$ for temperatures between $5{ }^{\circ} \mathrm{C}$ and $105^{\circ} \mathrm{C}$ and an EC uncertainty of $\pm 0.5 \%$ of the reading after temperature compensation. A data logger installed in the station was permanently recording the weather conditions: air temperature, relative humidity, solar radiation and rain precipitation. A Bland-Altman agreement analysis was also performed between the reference conductivity meter and the AS.

During the whole season, all conventional agricultural practices for the local rice cultivation were applied, such as surface fertilization and herbicide spraying.

\section{Results and discussion}

Fig. 11a and $\mathrm{b}$ show the linear regression and the Bland-Altman analysis made for the saline solutions measurements with $T_{\mathrm{w}}$ between $24.1{ }^{\circ} \mathrm{C}$ and $24.6^{\circ} \mathrm{C}$. This lab test shows full linearity $\left(R^{2}=1\right)$ between the handheld conductivity meter and the EC value obtained with the AS. The maximum standard deviation for the repeated measurements was $0.01 \mathrm{dS} \mathrm{m}^{-1}$ over the EC range from 0.50 to $5.00 \mathrm{dS} \mathrm{m}^{-1}$. This translates into a maximum uncertainty of $\pm 0.36 \%$ relative to the reading hence it is comparable to the uncertainty of the conductivity meter. On the other hand, the conductivity meter and the AS agree within $\pm 0.03 \mathrm{dS} \mathrm{m}^{-1}$ (95\% confidence level) with an offset of $-0.05 \mathrm{dS} \mathrm{m}^{-1}$.

The linear regression equation between the AS and the conductivity meter indicates that there is $1 \%$ gain deviation and an offset deviation of $-0.03 \mathrm{dS} \mathrm{m}^{-1}$ hence a two-point calibration with two reference conductivity solutions would correct the measurements. However, to detect $2 \mathrm{dS} \mathrm{m}^{-1}$ threshold, the AS was calibrated with a solution with $\mathrm{EC} \approx 2.00 \mathrm{dS} \mathrm{m}^{-1}$ (one-point calibration) before the field test.

Table 1 shows the measured EC for a reduced set of saline solutions and under different temperatures. The AS temperature measurements differ by less than $0.1{ }^{\circ} \mathrm{C}$ from those of the WTW conductivity meter. Also, the AS measures EC over the whole $T_{\mathrm{w}}$ range from $18{ }^{\circ} \mathrm{C}$ to $35^{\circ} \mathrm{C}$, with deviations similar to those found on the previous lab tests. The relative deviation ranged from $0.1 \%$ to $2.9 \%$ and is comparable to other experimental setups like (Seoane et al., 2008) with a deviation range from $0.34 \%$ to $2.49 \%$ obtained with the AD5933, or (Sanchez et al., 2013) with a deviation range from $0.3 \%$ to $4.2 \%$ obtained with the AFE4300. These designs, however, were based on commercial monolithic impedance analyzers that need an AFE and a MCU to be fully operative hence increasing circuit complexity, bill of materials, power consumption and board space.
Figs. 12 and 13 show the results of the validation field test that ran for 107 consecutive days, with no intervention on the AS. Again, the correlation factor was quite high $\left(R^{2}=0.937\right)$, thus indicating strong linearity between the AS and the reference conductivity meter. The Bland-Altman shows good agreement between both the AS and the meter, and the confidence interval was now $\pm 0.14 \mathrm{dS} \mathrm{m}^{-1}$. Air temperature as recorded by the meteorological data logger ranged from $15^{\circ} \mathrm{C}$ to $37^{\circ} \mathrm{C}$ hence no large deviations from the expected temperature range were observed.

The time series in Fig. 12 shows that the AS follows the WTW EC trend all of the time, but deviations become slightly larger starting on the 70th day. That deviation kept increasing until the end of the field test. One possible cause might be a deviation in the sensor temperature measurement that is used to compensate the EC readings. However, considering that EC decreases with a ratio of $2 \% / \mathrm{K}$, a deviation of $0.5^{\circ} \mathrm{C}$ introduces a deviation of just $0.01 \mathrm{dS} \mathrm{m}^{-1}$ at $\mathrm{EC}=1.00 \mathrm{dS} \mathrm{m}^{-1}$ hence $\mathrm{EC}$ is not perturbed by temperature deviations. A more likely cause is the intrusion of material inside the volume delimiter through its slits. This would have a net effect of increasing the cell factor that, in turn, yield a larger $R_{\mathrm{s}}$ thus making the apparent EC to be lower as seen in Fig. 11.

The AS proved robust against biofouling and wear. After the cultivation season was finished, lab tests determined that the cell constant deviated by less than $3 \%$ from the nominal $0.8 \mathrm{~cm}^{-1}$. Rinsing and reassembling returned the initial nominal value hence it is suspected that, to some degree, housing geometry was slightly affected, perhaps because of the thermal stress during the season. Therefore, maintenance can be highly reduced during a long-term deployment of the AS which is in contrast with other long-term monitoring systems like that in (O'Flynn et al., 2010) where regular sensor cleaning was necessary in order to keep sensor accuracy over a two-month test.

\section{Conclusions}

The electrical conductivity of water is accurately monitored during a cultivation season that lasted more than three months by means of a prototype autonomous sensor that measures the electrical impedance of water and consumed only $15.32 \mu \mathrm{A}$. The synchronous sampling technique implemented by means of a low-cost low-power MCU and a very simple analog front-end effectively measured the complex impedance of water as well as its temperature. The sensor housing was built from PVC tubes and fittings and includes a two-electrode conductivity cell with a cell constant of $0.8 \mathrm{~cm}^{-1}$. The electrodes used were two 316L stainless steel acorn nuts that are simple to assemble. The equivalent EC of water at $25{ }^{\circ} \mathrm{C}$ was derived from the real part of the electrical impedance of water at $83.3 \mathrm{kHz}$, cancelling out the deviations introduced by the electrode polarization impedance. 
Lab tests established that the EC uncertainty is $\pm 0.04 \mathrm{dS} \mathrm{m}^{-1}$ for the operating conditions of $0.5-5.0 \mathrm{dS} \mathrm{m}^{-1}$ and was unaffected by temperature. The AS was installed and left unattended for 107 consecutive days -more than any previously reported AS, to our knowledge- in a rice field and the EC readings showed deviations smaller than $\pm 0.14 \mathrm{dS} \mathrm{m}^{-1}$, making this design approach quite suitable for practical applications. The observed difference in accuracy with respect to the lab tests can be attributed to material intrusion inside the conductivity cell and to a deviation below $3 \%$ from the nominal cell constant of $0.8 \mathrm{~cm}^{-1}$. However, for practical purposes this deviation is adequate to detect an increase in water EC above $2 \mathrm{dS} \mathrm{m}^{-1}$.

\section{Declaration of Competing Interest}

None

\section{Acknowledgements}

This work has been funded by the European Commission grant No. 285861. The authors would like to thank the cooperation of Prof. Dimitris Katsantonis and Dr. Christos Dramalis for the field tests and technical assistance of Mr. Francis López.

\section{References}

Aliau-Bonet, C., Pallàs-Areny, R., 2013. Transient reduction in pulse-based impedance measurements. In: Proceedings of the 19th Symposium IMEKO TC 4. Barcelona, pp. $12-17$.

Grattan, S.R., 2002. Irrigation Water Salinity and Crop Production. Univ. Calif. Agric. Nat. Resour. 9.

Grattan, S.R., Shannon, M.C., Roberts, S.R., 2002. Rice is more sensitive to salinity than previously thought. Calif. Agric. 56, 189-195. https://doi.org/10.3733/ca. v056n06p189.

Hanson, B., Grattan, S.R., Fulton, A., 2006. Agricultural salinity and drainage. Regents of the University of California, Davis.

López Riquelme, J.A., Soto, F., Suardíaz, J., Sánchez, P., Iborra, A., Vera, J.A., 2009. Wireless Sensor Networks for precision horticulture in Southern Spain. Comput. Electron. Agric. 68, 25-35. https://doi.org/10.1016/j.compag.2009.04.006.

Min, M., Märtens, O., Parve, T., 2000. Lock-in measurement of bio-impedance variations. Measurement 27, 21-28. https://doi.org/10.1016/S0263-2241(99)00048-2.

Nam, W.H., Kim, T., Hong, E.M., Choi, J.Y., Kim, J.T., 2017. A Wireless Sensor Network (WSN) application for irrigation facilities management based on Information and Communication Technologies (ICTs). Comput. Electron. Agric. 143, 185-192. https://doi.org/10.1016/j.compag.2017.10.007.

O’Flynn, B., Regan, F., Lawlor, A., Wallace, J., Torres-Sanchez, J., Ó Mathuna, S.C., 2010. Experiences and recommendations in deploying a real time, water quality monitoring system. Meas. Sci. Technol. 21. https://doi.org/10.1088/0957-0233/21/12/124004. Article.

Oppenheim, A.V., Willsky, A.S., Hamid, N., 1997. Signals \& Systems, 2nd ed. PrenticeHall Inc.l, New Jersey.

Pallàs-Areny, R., Webster, J.G., 1993. Bioelectric impedance measurements using synchronous sampling. IEEE Trans. Biomed. Eng. 40, 824-829. https://doi.org/10.1109/ 10.238468 .

Reverter, F., Pallàs-Areny, R., 2005. Direct Sensor-to-Microcontroller Interface Circuits: Design and Characterization, 1st ed. Marcombo S.A, Barcelona.

Sanchez, B., Praveen, A., Bartolome, E., Soundarapandian, K., Bragos, R., 2013. Minimal implementation of an AFE4300-based spectrometer for electrical impedance spectroscopy measurements. J. Phys. Conf. Ser. 434, 012014. https://doi.org/10.1088/ 1742-6596/434/1/012014.

Seoane, F., Ferreira, J., Sanchéz, J.J., Bragós, R., 2008. An analog front-end enables electrical impedance spectroscopy system on-chip for biomedical applications. Physiol. Meas. 29, S267-S278. https://doi.org/10.1088/0967-3334/29/6/S23.

Zhuiykov, S., 2012. Solid-state sensors monitoring parameters of water quality for the next generation of wireless sensor networks. Sens. Actuat., B Chem. 161, 1-20. https://doi.org/10.1016/j.snb.2011.10.078.

Zia, H., Harris, N.R., Merrett, G.V., Rivers, M., Coles, N., 2013. The impact of agricultural activities on water quality: A case for collaborative catchment-scale management using integrated wireless sensor networks. Comput. Electron. Agric. 96, 126-138. https://doi.org/10.1016/j.compag.2013.05.001. 